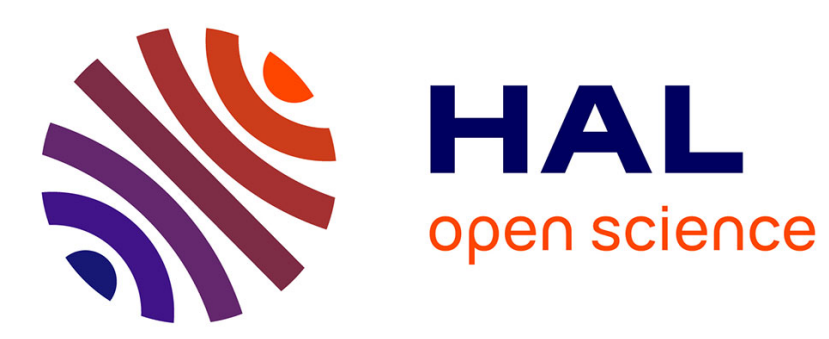

\title{
Multi-band supervised classification for polarimetric SAR
}

Xavier Dupuis, Valentine Wasik, Alexandre Alakian, Dominique Dubucq

\section{To cite this version:}

Xavier Dupuis, Valentine Wasik, Alexandre Alakian, Dominique Dubucq. Multi-band supervised classification for polarimetric SAR. IGARSS 2019, Jul 2019, YOKOHAMA, Japan. hal-02339219

\section{HAL Id: hal-02339219 \\ https://hal.science/hal-02339219}

Submitted on 30 Oct 2019

HAL is a multi-disciplinary open access archive for the deposit and dissemination of scientific research documents, whether they are published or not. The documents may come from teaching and research institutions in France or abroad, or from public or private research centers.
L'archive ouverte pluridisciplinaire HAL, est destinée au dépôt et à la diffusion de documents scientifiques de niveau recherche, publiés ou non, émanant des établissements d'enseignement et de recherche français ou étrangers, des laboratoires publics ou privés. 


\title{
MULTI-BAND SUPERVISED CLASSIFICATION FOR POLARIMETRIC SAR
}

\author{
Xavier Dupuis (1), Valentine Wasik (1), Alexandre Alakian (2), Dominique Dubucq (3) \\ (1) : DEMR, ONERA, F-13661 Salon cedex Air - France \\ (2) DTIS, ONERA, Université de Paris Saclay, F-91123 Palaiseau - France \\ (3) TOTAL S. A., Centre Scientifique et Technique Jean Féger, 64000 Pau - France.
}

\begin{abstract}
This work addresses the potential of multi-band polarimetric SAR imaging for terrains and vegetation classification. A classic supervised Wishart classifier is adapted to polarimetric multi-band datasets, and is applied on the X-, L- and UHF-band acquisitions done during the NAOMI campaign (ONERA-Total) in Gabon (Africa) in 2015. The contributions of the different frequencies are shown and discussed. It is shown that the use of the multi-band dataset improves significantly the classification result.
\end{abstract}

Index Terms - SAR, classification, multi-band

\section{INTRODUCTION}

The NAOMI project (New Advanced Observation Method Integration) is a Total and ONERA partnership initiated in 2014. Among the objectives of this project, one is to analyze the capacities of remote sensing techniques to characterize surfaces (biophysical and geological) and to detect hydrocarbons (directly or indirectly through environmental descriptors such as vegetation health and state). In this context, the NAOMI campaign that took place in Gabon in 2015 allowed ONERA and Total to construct a particularly diversified dataset composed by polarimetric SAR and VNIR-SWIR hyperspectral acquisitions along with in-situ measurements over the Mandji peninsula. This site presents an alternation between dense tropical forests, sparse and short vegetation, backwaters and man-made structures, with oil deposits that have been exploited for over 50 years in this area. On such equatorial area, optical acquisitions are difficult because of clouds and heavy rains season. Radar is a good alternative to overcome the weather problem. Moreover the swath of a SAR sensor is wider than optical one, which makes SAR imaging more suitable to cover large areas in such a place. Landscape classification from SAR imaging seems to be an interesting technique to characterize terrains and vegetation for the purpose of the NAOMI project. Moreover, the Mandji dataset gives us the opportunity to explore the contribution of several bands in the results of the classification products, since acquisitions have been done in X-, L- and UHF-band. In this work, we propose to adapt and apply a classic supervised Wishart classifier [1] to our multi-band SAR dataset. After a brief presentation of the data, the classifier methodology is explained. The reference regions from in-situ measurements are then detailed, and the classification results are finally discussed.

\section{DATASET}

Data were acquired with SETHI [1], an ONERA sensor, in Gabon over Mandji area in 2015 , July $8^{\text {th }}$. Two flights were performed, one to acquire simultaneously $\mathrm{X}$ and $\mathrm{L}$ bands and a second to acquire simultaneously UHF and L bands. X and UHF bands were acquired in full polarimetric mode. Lband was acquired in dual-polarimetric mode. Table 1 summarizes the characteristics of the data.

\begin{tabular}{|c|c|c|c|}
\hline SAR data & $\begin{array}{c}\text { UHF/VHF } \\
\text { band }\end{array}$ & L band & X band \\
\hline Bandwidth & $200 \mathrm{MHz}$ & $150 \mathrm{MHz}$ & $300 \mathrm{MHz}$ \\
\hline $\begin{array}{c}\text { Range } \\
\text { resolution } \\
\text { Azimuth } \\
\text { resolution }\end{array}$ & $0.66 \mathrm{~m}$ & $0.88 \mathrm{~m}$ & $0.44 \mathrm{~m}$ \\
\hline Windowing & rectangular & rectangular & rectangular \\
\hline Polarization & full & Dual Hh-Hv & full \\
\hline
\end{tabular}

Table 1: SAR dataset main characteristics.

Figure 1 to Figure 3 present the SAR images of the area at $\mathrm{X}$-band, L-band and UHF-band respectively.

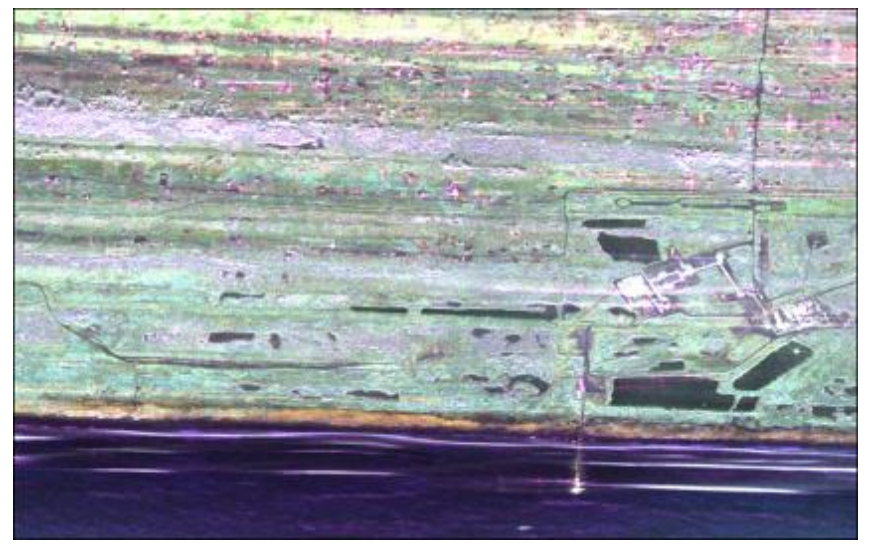


Figure 1: $\mathrm{X}$-band data, Hh polarization is coded on the Red channel, Vh on the Green channel and $\mathrm{Vv}$ on the Blue one.

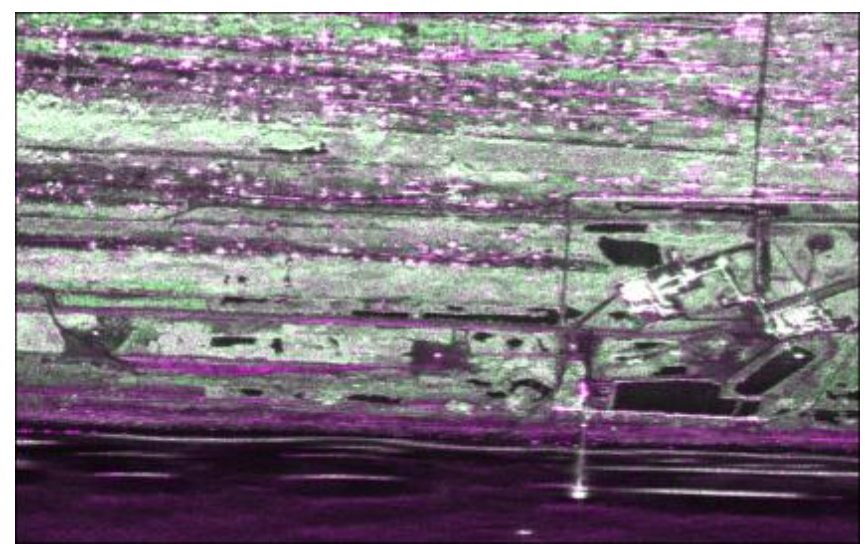

Figure 2: L-band data, Hh polarization is coded on the Red channel, Hv on the Green channel and $\mathrm{Hh}$ on the Blue one.

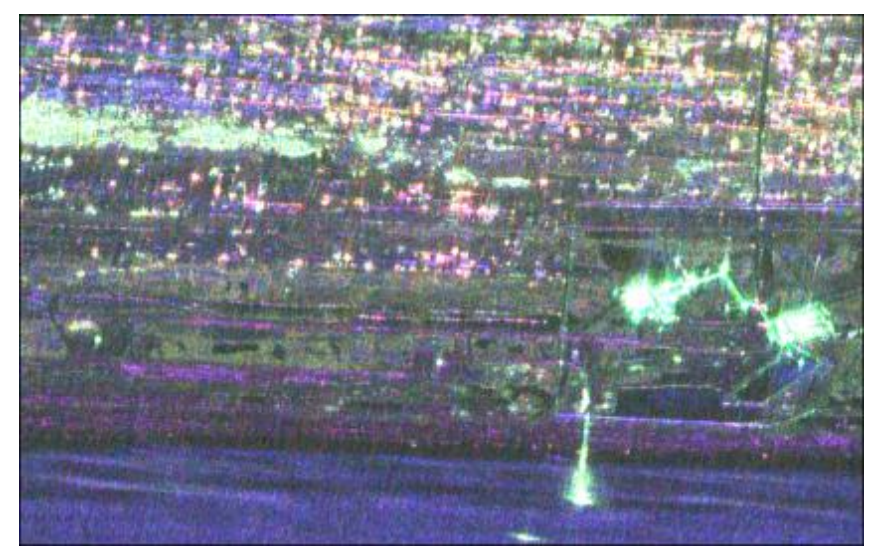

Figure 3: UHF-band data, Hh polarization is coded on the Red channel, Vh on the Green channel and Vv on the Blue one.

The SAR dataset is coregistered and projected into the Xband native slc geometry for classification purpose.

Flights dedicated to optical acquisitions were operated the same day. The characteristics of the hyperspectral cameras are presented in Table 2.

\begin{tabular}{|c|c|c|}
\hline Imaging & $\begin{array}{c}\text { Hyspex VNIR } \\
\text { FOV expander } \\
\text { Pushbroom }\end{array}$ & Hyspex SWIR \\
\hline Spatial pixels & 1600 & 320 \\
\hline Field of view & $34^{\circ}$ & $14^{\circ}$ \\
\hline Spectral range & $0.4-1 \mu \mathrm{m}$ & $1-2.5 \mu \mathrm{m}$ \\
\hline $\begin{array}{c}\text { Spectral bands } \\
\text { Spectral sampling } \\
\text { distance }\end{array}$ & 160 & 256 \\
\hline $\begin{array}{c}\text { Ground sampling } \\
\text { distance }\end{array}$ & $3.7 \mathrm{~nm}$ & $6 \mathrm{~nm}$ \\
\hline
\end{tabular}

Table 2 : Hyperspectral cameras characteristics.

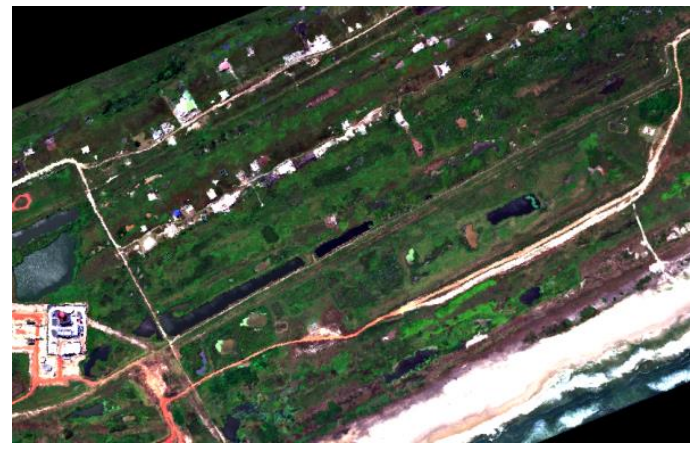

Figure 4 : Optical image of the Mandji site.

SAR and hyperspectral (VNIR, SWIR) data are groundcoregistered [3] in a common WGS84 referential.

\section{MULTIBANDS CLASSIFIER}

The Wishart classifier is an interesting and largely used technique for the classification of the landscapes with polarimetric SAR images [2]. This iterative method focuses on the statistical properties of the response. For fullypolarimetric SAR data, the complex response for each pixel can be written in the Pauli vector $\boldsymbol{k}=\frac{1}{\sqrt{2}}\left[S_{H h}+S_{V v}, S_{H h}-\right.$ $\left.S_{V v}, 2 S_{H v}\right]^{T}$, where $S_{X y}$ is the complex backscattered signal for an emitted polarization $X$ and a direction of reception $y$ $(X=H, V ; y=h, v)$. In case of fully-developed speckle, $\boldsymbol{k}$ is assumed to be a random vector following a complex circular multi-variate Gaussian law with zero-mean for which the statistical properties are entirely described by its covariance matrix $\mathbf{T}=\left\langle\boldsymbol{k} \boldsymbol{k}^{\dagger}\right\rangle$. For a set of $N$ measurements $\left\{\boldsymbol{k}_{1}, \ldots, \boldsymbol{k}_{N}\right\}$, the maximum-likelihood estimator for $\mathbf{T}$ (ie $\widehat{\mathbf{T}}=\frac{1}{N} \sum_{n=1}^{N} k_{n} k_{n}^{\dagger}$ ) follows a complex Wishart law [1]. The principle of the Wishart classifier is to use this property to construct a measure for classification. For each pixel of the image and its associated empirical covariance matrix $\widehat{\mathbf{T}}$, the following measure is calculated for each class $w_{i}$ [1]: $d_{W}\left(\widehat{\mathbf{T}}, w_{i}\right)=\ln \left|\mathbf{V}_{i}\right|+\operatorname{tr}\left(\mathbf{V}_{i}^{-1} \widehat{\mathbf{T}}\right)$, where $V_{i}$ is the covariance matrix of the pixels in the class (i.e. class center), |. | is the determinant, and $\operatorname{tr}($.$) is the trace operator. The pixel is$ assigned to the class $w_{j}$ for which the Wishart measure is the smallest. This algorithm is iterative. In our study the initialization is based on the supervised definition of the classes and for each step the class centers are updated. The algorithm is stopped after 5 iterations.

In the Mandji data presented, the acquisitions have been done with three different frequency bands: X, L and UHF. The enhanced behaviors of the scene will be different from one frequency band to the other since the interaction between the media and the illuminating wave is very frequency dependent. The contribution of this frequency 
diversity in the classification process is an interesting point to focus on. For this purpose, we have modified the Wishart classifier so that multi-band polarimetric SAR data could be processed. For each pixel, the idea is to estimate one covariance matrix for each considered band $\left(\widehat{\mathbf{T}}^{X}, \widehat{\mathbf{T}}^{L}, \widehat{\mathbf{T}}^{U H F}\right)$ and to calculate independently the associated Wishart measures for a common ensemble of classes. The pixel is attributed to the class $w_{j}$ that minimizes the sum of the three measures:

$$
w_{j}=\min _{i}\left(d_{w}^{X}\left(\widehat{\mathbf{T}}^{X}, w_{i}\right)+d_{w}^{L}\left(\widehat{\mathbf{T}}^{L}, w_{i}\right)+d_{w}^{U H F}\left(\widehat{\mathbf{T}}^{U H F}, w_{i}\right)\right) .
$$

The algorithm is then iterated as previously.

One difficulty comes from the fact that the L-band acquisitions are dual-polarized (emission in $H$ only). To overcome this limitation, the coefficient $S_{V v}^{L}$ in the Pauli vector for the L-band is replaced by the ratio $S_{H v}^{L} / S_{H h}^{L}$.

\section{GROUND TRUTH}

Ground truth is based on in-situ photography and ASD reflectance ground measurements. These measurements are used to identify the classes, but they do not cover a sufficient area to get a reference ground truth map for the classification (see Figure 5 (a)). To extend the ground truth map, a classification on both VNIR and SWIR data taking into account the shape similarities of spectral reflectance (spectral angle), the reflectance amplitude similarities and the specific absorption is performed. The spectral angle criterion is the most sensitive parameter, a strict constraint is $1^{\circ}$ threshold and less strict is $3^{\circ}$. The results are presented in Figure 5.

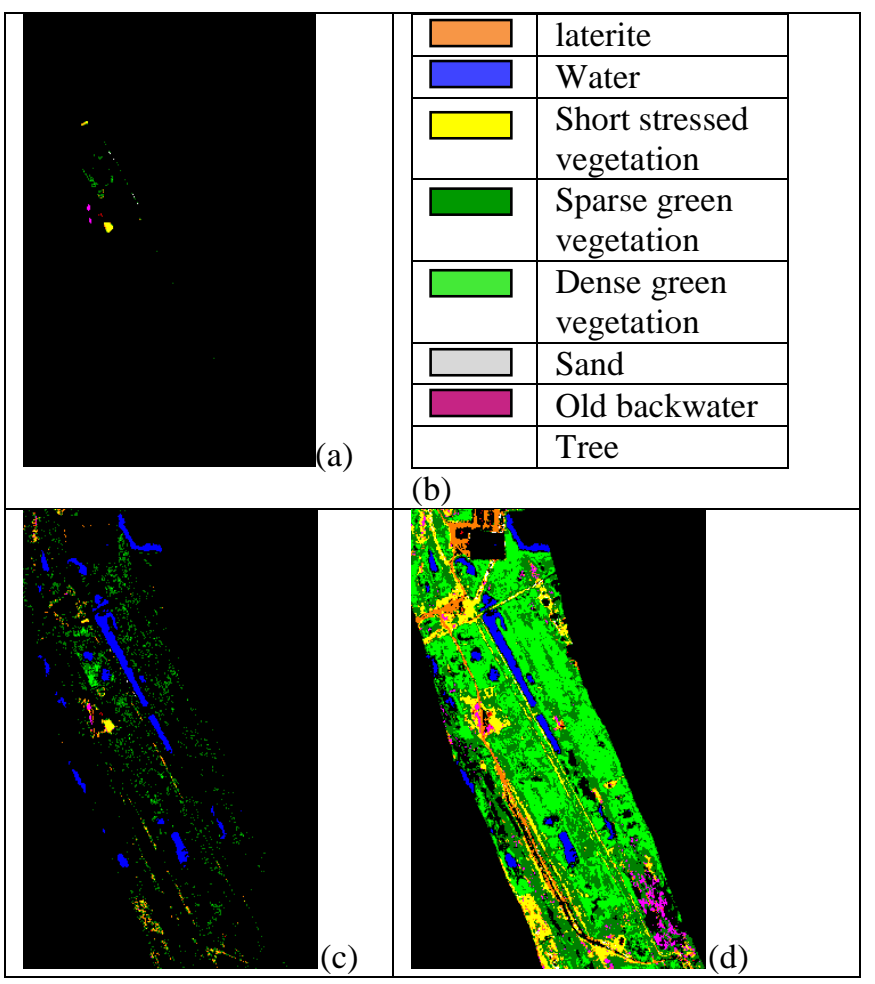

Figure 5 : Ground truth map : (a) in-situ ground truth (from ASD measurement), (b) classes definition, (c) computed map with $1^{\circ}$ spectral angle threshold and (d) computed map with $3^{\circ}$ spectral angle threshold.

\section{SAR CLASSIFICATION RESULTS}

Prior to the classification, a bilateral filtering is processed on each SAR images to attenuate the speckle noise [4]. The classification algorithm studied in this paper is supervised, so we first define the reference classes based on the extended ground truth map (Figure 5 (c)). The "old backwater" class was not considered for SAR classification since it was not sufficiently different from other classes. A class of "shadow" (colored in black in the following) has been added due to the particularity of the SAR acquisition geometry as well as a class for "man-made structures" in red A classification is performed for each band independently (X, L, and UHF) to analyze the impact of each radar band on the results. Then multi-band classifications are processed for "X plus L" and "X plus L plus UHF" combinations. Note that all the results presented below are based on the same class definition.

Figure 6 presents the classification result for X-band dataset. It appears noisy because $\mathrm{X}$-band is very sensitive to roughness of small objects, accordingly to its wavelength, and cannot distinguish vegetation types as trees, dense and short vegetation. At the opposite, the water (sea and backwater in blue), and the sand (in grey) are well detected.

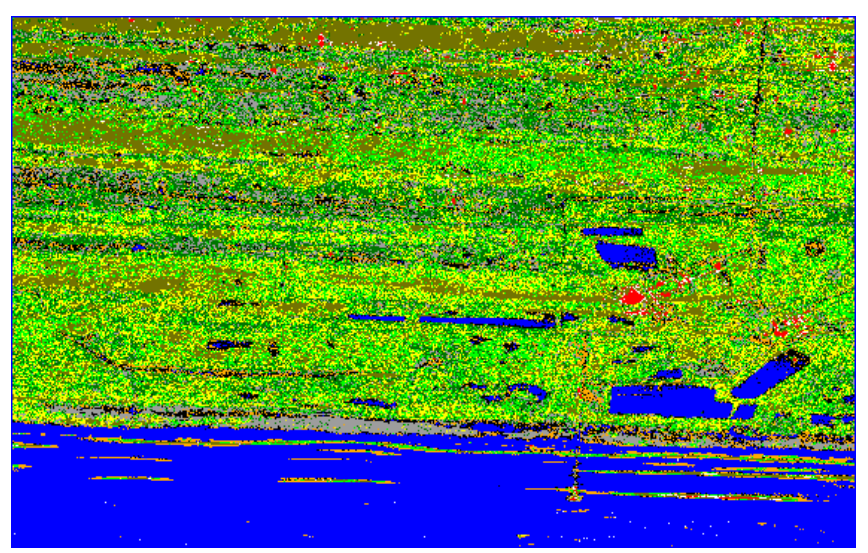

Figure 6 : X-band classification.

Figure 7 presents the L-band classification result. Compared to X-band, there are confusions for water (sea and backwater) but vegetation is less noisy except for the trees (dark green).

Figure 8 presents the UHF classification result. Except for the strong echoes from buildings and trees (red class) and for the sand on the beach, the classification is very noisy due to non-sensitivity of such a wavelength to short and medium vegetation. 


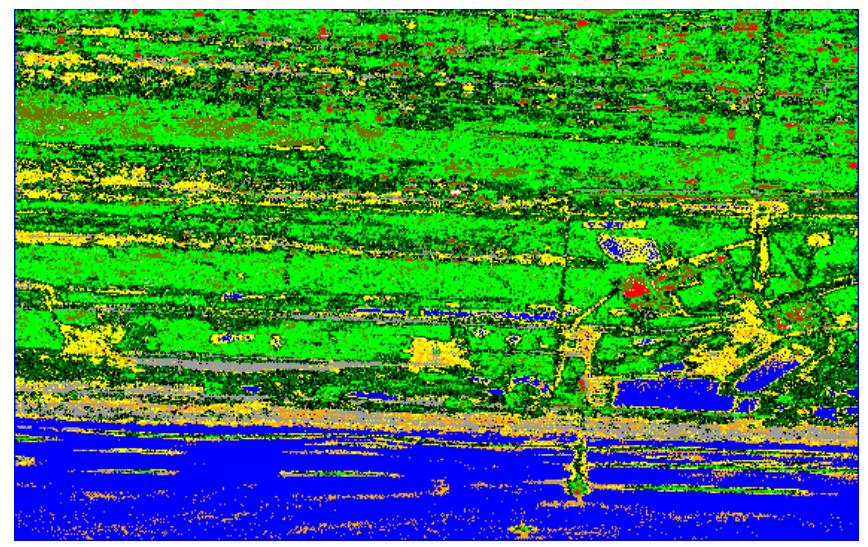

Figure 7 : L-band classification.

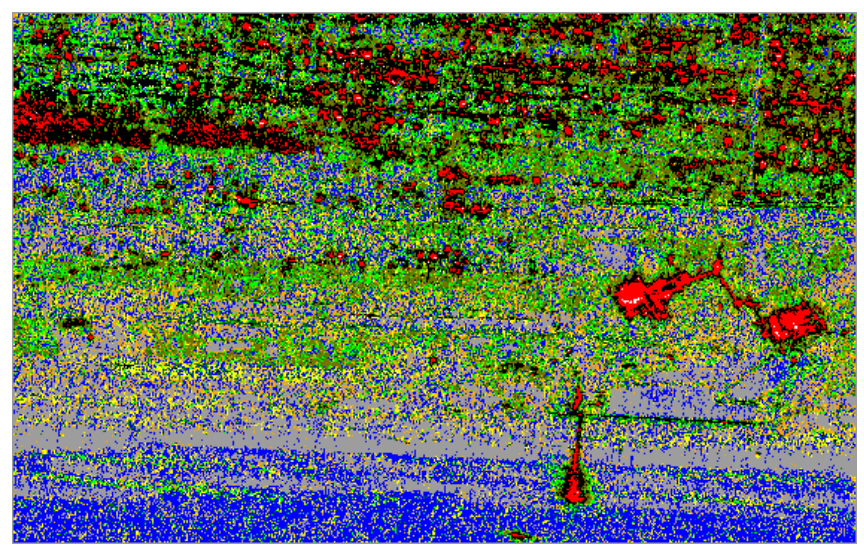

Figure 8 : UHF-band classification.

A first result of multi-band combination is presented Figure 9. Qualitatively, the "X-plus L-"band classification seems to provide a good result as it takes advantages of both bands. The sea and backwaters appears qualitatively well classified; the different classes of vegetation seem close to the extended ground truth map of Figure 5 (d). But the buildings (in red) and the trees (in the white ellipse) are not very well delimited.

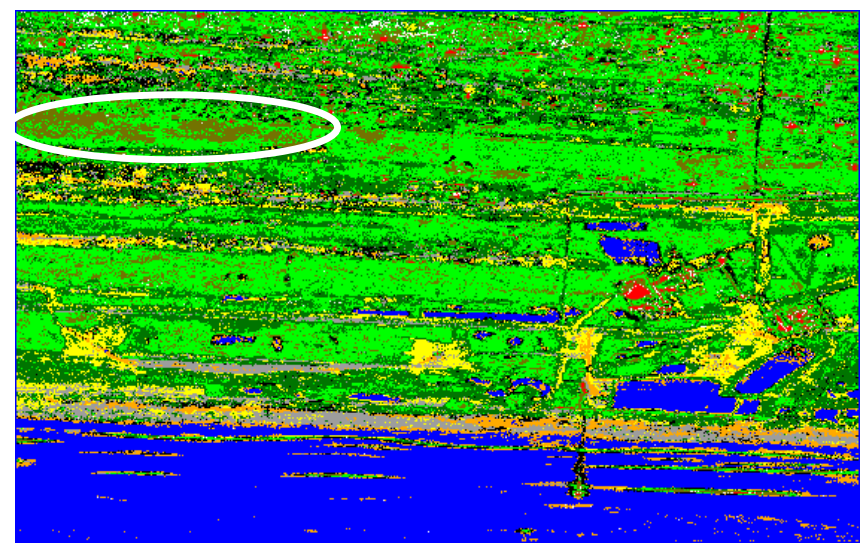

Figure $9: \mathrm{X}+\mathrm{L}$ band classification.
As it can be seen on Figure 10, combining the three bands, $\mathrm{X}, \mathrm{L}$ and UHF, improves significantly the classification of tall vegetation (in the white ellipse) and building (in red). This result takes advantage of the different bands: X-band for water and bare surfaces, L-band for short and medium vegetation and UHF-band for tall vegetation and buildings.

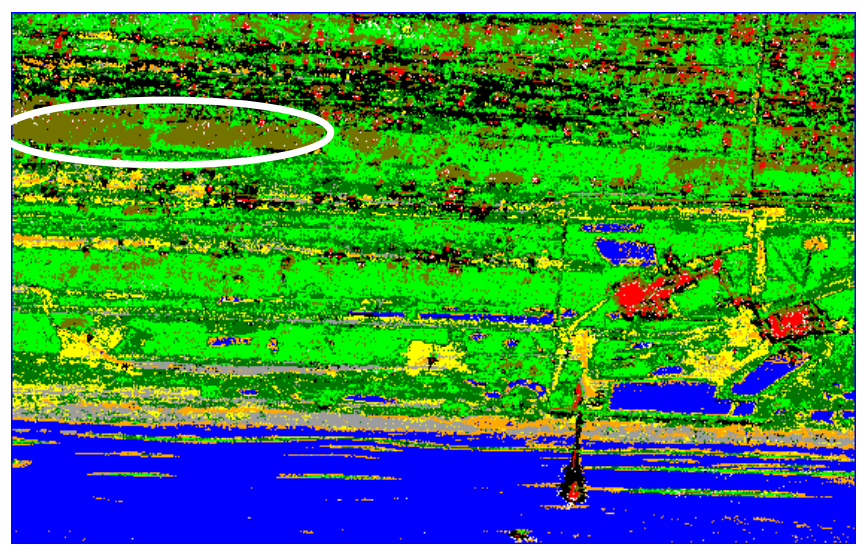

Figure $10: \mathrm{X}+\mathrm{L}+\mathrm{UHF}$ band classification.

\section{CONCLUSION}

For the Mandji dataset acquired by ONERA during the NAOMI campaign, multi-band SAR classification improves significantly the results for vegetated areas with different height and state of vegetation in comparison with using only mono-band data. It takes advantages form the different wavelength.

To complete this first qualitative analysis, a quantitative study with confusion matrices is going to be processed.

\section{REFERENCES}

[1] R. Baqué and al., SETHI / RAMSES-NG New performances of the flexible multi-spectral airborne remote sensing research platform, RADAR 2017, Belfast, 2017 October 23-26.

[2] J. S. Lee, M. R. Grunes, and K. Ri. (1994). Classification of multi-look polarimetric SAR imagery based on complex Wishart distribution, International Journal of Remote Sensing, 15(11), p.2299-2311.

[3] A. Plyer, et al. (2015). A New Coregistration Algorithm for Recent Applications on Urban SAR Images, Geoscience and Remote Sensing Letters, IEEE, 12(11), p.2198-2202.

[4] C. Tomasi, and R. Manduchi. (1998). Bilateral filtering for gray and color images, in Proc. 6th ICCV, Bombay, India, p.839. 